\title{
Introduction. Humanités numériques et analyses spatiales : enjeux et perspectives
}

Introduction. Digital Humanities and Spatial Analysis: Challenges and

Perspectives

Carmen Brando, Francesca Frontini, Dominic Moreau, Mathieu Roche et Éric Masson

\section{OpenEdition}

\section{Journals}

Édition électronique

URL : https://journals.openedition.org/revuehn/2038

DOI : 10.4000/revuehn.2038

ISSN : 2736-2337

Éditeur

Humanistica

Référence électronique

Carmen Brando, Francesca Frontini, Dominic Moreau, Mathieu Roche et Éric Masson « Introduction.

Humanités numériques et analyses spatiales : enjeux et perspectives», Humanités numériques [En

ligne], 3 | 2021, mis en ligne le 01 mai 2021, consulté le 15 juillet 2021. URL : http://

journals.openedition.org/revuehn/2038; DOI : https://doi.org/10.4000/revuehn.2038

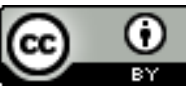

Les contenus de la revue Humanités numériques sont mis à disposition selon les termes de la Licence Creative Commons Attribution 4.0 International. 


\title{
humanités \\ numériques
}

3 | 2021

Humanités numériques spatialisées

\section{Introduction. Humanités numériques et analyses spatiales : enjeux et perspectives Introduction. Digital Humanities and Spatial Analysis: Challenges and Perspectives}

\author{
Carmen Brando, Francesca Frontini, Dominic Moreau, \\ Mathieu Roche et Éric Masson
}

\section{Résumés}

Cet article propose une introduction aux contributions présentées dans ce numéro thématique dédié aux humanités numériques spatialisées. Trois grands thèmes sont abordés : (1) le traitement de l'information spatiale dans des corpus textuels issus des travaux en sciences humaines et sociales, principalement en études littéraires ; (2) les problématiques d'acquisition, de spatialisation et de diffusion de données géographiques du passé et du patrimoine, donc, ici, davantage en lien avec la recherche en histoire ; (3) l'information spatiale, son traitement et ses usages en archéologie. Pour chacun de ces thèmes, nous présentons les initiatives fondatrices, un bref état de l'art et une synthèse des contributions.

This paper provides an introduction to the contributions presented in this thematic issue dedicated to the spatial humanities. Three main themes are addressed: (1) the processing of spatial information in textual corpora resulting from work in the human and social sciences, mainly in literary studies; (2) problems of acquisition, spatialisation and dissemination of geographical data of the past and from cultural heritage, thus, here, more connected with research in history; (3) spatial information and its processing and uses in archaeology. For each of these topics, we present the founding initiatives with historiographical elements, a brief status quaestionis and a synthesis of the contributions. 
Este artículo ofrece una introducción a los principales temas identificados en las contribuciones presentadas en este número temático dedicado a las humanidades digitales espaciales. Hemos identificado tres temas que se enumeran a continuación: (1) el tratamiento de la información espacial en los corpus textuales resultantes de trabajos en humanidades y ciencias sociales, con una importante presencia de los estudios literarios; (2) los problemas de adquisición, espacialización y difusión de los datos geográficos del pasado y del patrimonio, a menudo relacionados con la investigación en historia; (3) la información espacial, su tratamiento y usos, en arqueología. Para cada tema, presentamos las iniciativas fundadoras con elementos historiográficos, un breve estado de la cuestión y una síntesis de las contribuciones.

\section{Entrées d'index}

MOTS-CLÉS : humanités numériques spatialisées, archéologie, histoire, histoire des humanités numériques, système d'information géographique, cartographie, analyse spatiale, analyse textuelle KEYWORDS: spatial digital humanities, archaeology, history, history of the digital humanities, geographic information system, cartography, spatial analysis, textual analysis

Depuis les travaux fondateurs de John Snow (1813-1858) sur l'analyse spatiale (Snow 1855) et de Charles Joseph Minard (1781-1870) sur la représentation des données spatiotemporelles (Minard 1869), ou encore ceux de Georg Simmel (1858-1918) et de la première école de Chicago, jusqu'à ceux de chercheurs plus proches de nous comme Milton Santos (19262001), Isaac Joseph (1943-2004) ou Jacques Lévy (1952-), en passant par l'œuvre de Le Corbusier (1887-1965), l'analyse des informations spatiales constitue un prisme fondamental d'approche et de recherche dans les sciences humaines et sociales (SHS). Le basculement progressif dans le numérique (deuxième moitié $\mathrm{du} \mathrm{xx}^{\mathrm{e}}$ siècle) et dans l'usage d'Internet (fin $\mathrm{du} \mathrm{xx}^{\mathrm{e}}$ siècle), appliqués aux sciences humaines et sociales, va permettre l'émergence et le déploiement des humanités numériques (HN) telles qu'on les pratique aujourd'hui. À partir des années 1970, par exemple, les systèmes d'information géographique (SIG) progressent et se propagent depuis la géographie numérique vers les disciplines connexes (histoire, archéologie, etc.), démontrant ainsi l'intérêt et l'utilité de l'analyse spatiale et de son intégration dans la «boîte à outils " des praticiens du numérique en SHS. De son côté, la communauté des humanités numériques montre un fort intérêt vers les questions liées à l'espace, sa modélisation et sa représentation ${ }^{1}$; l'Alliance of Digital Humanities Organizations assure ainsi l'animation de plusieurs groupes d'intérêts, dont Geohumanities ${ }^{2}$, qui recense et promeut les projets en humanités numériques appuyés sur l'usage des SIG et de la géovisualisation, privilégiant des approches spatiales, spatiotemporelles et localisées.

Aujourd'hui, les méthodes informatiques vont au-delà de la "simple " géovisualisation des données sous un angle SIG, en proposant des techniques issues du traitement automatique du langage naturel (TALN), des sciences des données, de l'intelligence artificielle (apprentissage ma- 
chine et profond) et du Web des données (Linked Data), qui permettent d'explorer de nouvelles perspectives spatiales et spatiotemporelles de l'information en SHS. L'ouvrage de Bodenhamer, Corrigan et Harris (2010) ou l'article de Murrieta-Flores et Martins (2019), pour ne citer que quelques travaux fondateurs des humanités numériques spatialisées dans le monde anglo-saxon, vont dans cette direction.

Le présent numéro thématique de la revue Humanités numériques s'inscrit lui aussi dans cette optique. En amont de sa composition, deux événements scientifiques organisés en 2018 ont permis de réunir les éditeurs invités : un atelier traitant de la problématique des humanités numériques spatialisées, à Montpellier ${ }^{3}$, et deux journées d'étude exploratoires "Archéologie, histoire et analyse spatiale ", à Lille ${ }^{4}$. Les contributions qui composent ce volume ont alors été sélectionnées suite aux présentations réalisées lors de ces deux événements et enrichies à travers un appel à communications ouvert ${ }^{5}$. Tous les textes ont été évalués conformément aux principes de la revue. Finalement, les différentes contributions retenues pour ce numéro spécial se déclinent selon les trois grands thèmes détaillés dans les sections suivantes.

\section{Information spatiale et corpus textuels}

Les textes occupent une place majeure dans un grand nombre de disciplines en SHS et de plus en plus de travaux de recherche constituent des corpus textuels afin de les analyser en s'appuyant sur la statistique textuelle (par exemple, occurrences, cooccurrences) et la linguistique de corpus (via un concordancier, par exemple). De même, l'analyse d'un corpus par son annotation, autrement dit, par un procédé consistant à ajouter des couches d'informations structurées au texte, permet d'élargir les possibilités d'interrogation et d'analyse de corpus. Cette pratique d'annotation est largement répandue dans les HN notamment dans le contexte de la norme TEI (Text Encoding Initiative) grâce à laquelle les textes sont encodés à partir de jeux de balises XML définies et décrites par le consortium TEI. Dans la réflexion autour des éléments susceptibles d'être annotés et référencés en TEI, les éléments spatiaux et l'information géographique occupent une place importante ${ }^{6}$ (voir entre autres Frontini et al. 2016).

En littérature, le premier à mener une étude exhaustive sur l'espace et le récit littéraire sera Mikhaïl Bakhtine (1978) dans son essai sur le chronotope, dans lequel il théorise des modes de représentation de l'espace et du temps dans le langage et le discours. Plus tard, Franco Moretti (200o, 2007) suivra et s'intéressera aux liens entre la géographie et la littérature, et définira sa célèbre méthode pour la lecture distante (distant reading) de textes littéraires ; son atlas (Moretti 1999) rassemblera ainsi une centaine de cartes élaborées à partir d'un large corpus de romans européens. Les travaux de Bakhtine et de Moretti ont inspiré par la suite un important nombre d'études qui mobilisent des experts en littérature, des historiens, des géographes et des géomaticiens ainsi que des linguistes et des informaticiens, comme celle de Piatti et al. (2009) plaidant pour une cartographie littéraire de la fiction, et celle de Aron et al. (2017) qui propose une analyse chronotopique des Mystères de Bruxelles (1845-1846). Dans ce cadre, le projet Mapping the Lake District ${ }^{7}$ (Donaldson, Gregory 
et Murrieta-Flores 2015) reste précurseur de la méthode dite d'analyse spatiale textuelle visant à annoter, géoréférencer et analyser un texte par la linguistique outillée. L'aspect novateur de ce projet est d'analyser non seulement les lieux cités dans un recueil de récits de voyage des écrivains anglais dans le Lake District (nord-ouest de l'Angleterre), un lieu central dans le panorama littéraire du romantisme britannique, mais aussi leur contexte linguistique, pour en étudier la perception.

Toute entreprise de spatialisation de corpus textuels en vue d'une cartographie et d'une analyse spatiale doit s'intéresser à la manière dont les lieux et toutes autres références spatiales sont désignés dans les données textuelles. Cela implique de définir la notion de lieu de manière formelle tout en s'intéressant à ses différents contextes d'utilisation dans les textes. Une autre problématique associée s'intéresse à traduire les informations de localisation (les noms de lieux et relations spatiales) sous forme de coordonnées. Dans les processus d'identification de références spatiales à partir de textes, le TALN, discipline mobilisant des approches de l'informatique, de la linguistique et des sciences cognitives, peut être appliqué. En général, l'identification automatique d'un nom de lieu comprend deux étapes : la reconnaissance des entités nommées (REN) et la désambiguïsation de ces dernières par l'attribution d'un identifiant (NEL, pour Named Entity Linking). Cette dernière étape permet notamment d'obtenir des coordonnées de localisation par le biais de référentiels géographiques sémantisés mis à disposition aujourd'hui dans le Web des données. Pour les références spatiales dans les textes véhiculant des lieux qui ont changé de nom, de nature ou de localisation, la question du référentiel géohistorique à utiliser reste une problématique difficile.

Il existe de nombreuses approches de TALN pour résoudre le problème d'identification de références spatiales dans un texte. Dans le contexte des HN, nous pouvons citer les travaux de Moncla et al. (2016) pour reconstruire des itinéraires à partir des récits historiques de voyages, de Frontini et al. (2016) pour spatialiser un corpus d'essais de critique littéraire des auteurs français du XIX ${ }^{\mathrm{e}}$ siècle, ou encore de McDonough, Moncla et van de Camp (2019) pour identifier et localiser automatiquement les noms de lieux cités dans l'Encyclopédie de Diderot et d'Alembert. Selon les textes à analyser, ces méthodes nécessitent d'être adaptées pour les rendre pertinentes pour une analyse. Cela implique de modifier ou de créer des ressources spécifiques (textes annotés, dictionnaires) pour la REN et le NEL. Il existe des outils pour faciliter le travail d'annotation manuelle des entités nommées dans un texte et, dans le but d'enrichir une édition numérique encodée selon les normes de la TEI, Recogito ${ }^{8}$ (Simon et al. 2015) est sans doute l'un des plus utilisés par la communauté des HN ; les spécialistes de l'Antiquité s'en sont particulièrement emparés. Cette plateforme intègre désormais des méthodes de REN pour certaines langues pour lier les entités nommées à des référentiels géohistoriques sémantisés, ce qui permet de contextualiser l'entité dans l'espace et dans le temps ainsi que d'attester de son existence d'après une source historique. Dans le contexte de la norme TEI, il est enfin important de citer la réflexion menée au sein du consortium CAHIER ${ }^{9}$ de la TGIR Huma-Num sur l'harmonisation des pratiques d'annotation des références spatiales dans les corpus d'auteurs. 
Dans la perspective d'analyse littéraire, l'action COST Distant Reading for European Literary History ${ }^{10}$ met l'accent sur l'utilisation de la lecture distante et constitue un corpus homogène et en plusieurs langues européennes, composé de textes de romans européens de l'époque moderne. La compilation qui en résulte, nommée ELTeC ${ }^{11}$ (Corpus multilingue de littérature européen), a été annotée en entités spatiales en s'appuyant sur un guide d'annotation défini. Ce travail a pour ambition la constitution d'un corpus annoté de manière homogène permettant une évaluation des systèmes REN de l'état de l'art et l'annotation de textes littéraires (Frontini et al. 2020). Au-delà de références spatiales, d'autres travaux s'intéressent à l'identification et la cartographie des opinions et des émotions véhiculées par les lieux dans les textes (Kergosien et al. 2015 ; Dominguès et al. 2019).

Dans la première partie de ce numéro, quatre contributions s'intéressent aux problématiques précédemment mentionnées, à savoir l'annotation de références spatiales par la mobilisation de méthodes de TALN et d'encodage selon les normes TEI. Anne Klammt décrit les travaux de modélisation spatiale en TEI de grandes bases de données textuelles du Centre allemand d'histoire de l'art (DFK) à Paris. Ioana Galleron, Roxana Patraș, Camelia Grădinaru et Frédérique Mélanie-Becquet proposent une réflexion sur la spatialisation des textes à l'issue d'une campagne d'annotation manuelle des entités spatiales dans un corpus de romans roumains du XIX ${ }^{\mathrm{e}}$ siècle. Motasem Alrahabi, Carmen Brando, Muhamed AlKhalil et Joseph Dichy présentent une approche de TALN pour annoter les modalités sémantiques positives ou négatives associées aux noms de lieux dans des textes en arabe, en s'appuyant sur les SIG pour proposer des cartographies thématiques. Enfin, la contribution d'Hélène Flamein et Iris Eshkol-Taravella décrit une approche de TALN pour extraire des références spatiales dans le corpus Enquêtes sociolinguistiques à Orléans (ESLO).

Sur le plan des ressources, ces quatre contributions portent sur des langues, époques, modalités textuelles différentes : les descriptions de Paris dans les récits de voyage d'écrivains arabes ; les romans des haïdouks, hors-la-loi roumains ; les récits de voyageurs allemands à l'époque baroque ; les corpus oraux transcrits des enquêtes sociolinguistiques menées en France. Les objectifs de ces études sont complémentaires en s'intéressant à l'annotation automatique, à l'évaluation des outils disponibles et aux consignes d'annotation des données textuelles. Ces travaux sont par ailleurs parfaitement représentatifs des problématiques de recherche actuelles, en croisant des approches de TALN dans le cadre des HN. 


\section{Intégration et usages des données historiques et patrimoniales}

Le deuxième grand thème traité dans ce numéro porte sur des questions-clés et récurrentes en SIG historique, comme l'acquisition des données géographiques à partir des plans anciens ou l'analyse géohistorique du passé des villes à partir de sources historiques et hétérogènes. Elle aborde aussi de nouvelles problématiques, comme l'enrichissement et la diffusion de référentiels géohistoriques ou patrimoniaux conformes aux standards et technologies du Web des données. Les sciences historiques participent activement depuis plus de 25 années au déploiement des humanités numériques sous l'impulsion de chercheurs tels que Roy Rosenzweig (1950-2007) qui fonda en 1994 le Center for History and New Media dans le département d'histoire et d'histoire de l'art à l'université George Mason (Fairfax, Virginie, États-Unis ${ }^{12}$ ).

La dynamique des initiatives internationales autour des humanités numériques spatialisées explique le lien et l'engouement déjà vieux de quelques décennies entre l'histoire et la géomatique (Knowles 2000) au sein des HN. Ce rapprochement s'est progressivement mis en place autour de trois principaux types de collaboration bidisciplinaires entre : (1) les historiens et les géographes, en parallèle de l'évolution des outils de cartographie numérique (CAO) et des systèmes d'information géographique (SIG) ; (2) les historiens et les documentalistes, en parallèle de la numérisation des archives et de la construction des bases de données documentaires accessibles en ligne ; (3) entre les historiens et les archéologues, en parallèle de l'évolution des outils et des méthodes numériques de restitution utilisés pour visualiser, archiver et analyser les données spatiotemporelles mises à jour sur les chantiers de fouilles. Représentation spatiale numérique et gestion de bases de données historiques ont ainsi progressivement intégré les outils et méthodes de la géomatique dans les sciences historiques depuis le milieu des années 1980.

Les SIG purement historiques sont aujourd'hui nombreux (même s'il y en a nettement moins pour l'Antiquité et le Moyen Âge que pour les autres périodes) et, sans vouloir être exhaustif, nous pouvons citer quelques travaux récents et remarquables comme ceux de Bretagnolle, Giraud et Verdier (2010) qui modélisent l'efficacité du réseau des routes postales dans la France préindustrielle, ceux de Varet-Vitu, Marraud et Mermet (2020) qui analysent spatialement les professions et les activités des Parisiens à la veille de la Révolution, ou encore ceux de Pinol (2019) sur la déportation des Juifs de France et de Bernard et al. (2012). Du point de vue théorique, mentionnons l'ouvrage de Gregory et Ell (2007) qui propose une vue complète des usages du SIG en histoire ainsi qu'une critique de leur impact dans les pratiques des historiens, notamment en histoire urbaine.

Dans le contexte français, évoquer le SIG historique revient immanquablement à mentionner le programme de recherche ALPAGE (Noizet, Bove et Costa 2013), qui est l'un des précurseurs d'une analyse géomatique et diachronique d'un espace géographique. Ce projet mené entre 2006 et 2010 porte précisément sur l'espace parisien, avec pour 
principal objectif « de mettre en place des outils de travail mutualisés STIC-SHS permettant de développer les recherches concernant l'espace urbain parisien, grâce notamment à un SIG comprenant des couches cadastrales et historiques [...] de capitaliser une information robuste, susceptible de devenir un matériau réutilisable par tous, lors de recherches ultérieures ${ }^{13}$ \%. Ce succès pluridisciplinaire de reconstitution géomatique et historique se poursuit aujourd'hui par le consortium Paris Time Machine (PTM) de la TGIR Huma-Num, dont le principal objectif est « la constitution d'un référentiel géohistorique [qui] doit permettre à des chercheurs de différentes disciplines historiques de travailler ensemble sur un espace géographique commun, en l'occurrence, la région parisienne ${ }^{14}$ ».

La numérisation en 2000 et la diffusion par la BNF de la couverture numérique nationale de la carte de Cassini est un échelon important dans le développement du SIG historique en France. L'assemblage de l'ensemble des planches et leur géoréférencement ont été assurés par le LaDéHiS ${ }^{15}$ à l'EHESS ${ }^{16}$. L'IGN a ensuite contribué dans la mise à disposition de ces données dans le Géoportail en 2013. Aujourd'hui, le travail de vectorisation de la carte Cassini est assuré par l'équipe GeoHistoricalData ${ }^{17}$ composée par des chercheurs de l'EHESS et de l'IGN grâce à une approche de SIG collaboratif, ce qui a permis notamment la diffusion du référentiel géohistorique des villes et routes du Royaume de France (Perret, Gribaudi et Barthelemy 2015 ; Dumenieu et al. 2019). Aussi, la base de données en ligne dite Cassini ${ }^{18}$, qui recense l'historique des communes de France ainsi que l'évolution de la population, est une ressource essentielle en démographie historique. Plus récemment, la carte géoréférencée de Cassini a été utilisée pour lier les données de l'historique des communes avec les lieux nommés de la carte. Ce travail a abouti à la construction d'une base géographique et démographique, à l'échelle du " clocher ", qui recense l'intégralité des paroisses, hameaux ou maisons religieuses devenus plus tard chefs-lieux de commune, avec leur population de 1793 à $1999^{19}$.

Le chantier numérique Cassini et d'autres fonds cartographiques nationaux qui ont suivi (carte d'état-major du XIX ${ }^{\mathrm{e}}$ siècle, cartes topographiques au 1/80 ooo, 1/50 ooo et $1 / 25$ ooo) est un projet évolutif, de portée nationale, permettant aux historiens de croiser les données des époques modernes ou contemporaines avec des fonds de cartes, des localisations ou des photographies aériennes (pour le $\mathrm{xx}^{\mathrm{e}}$ siècle) sur l'ensemble du territoire métropolitain. Ce travail de vectorisation de fonds anciens permet l'émergence de référentiels géohistoriques (Costa 2012) ainsi que le développement des applications spécialisées pour les historiens. En particulier, le géocodage historique des adresses de villes est un besoin récurrent en histoire et des outils sont développés aujourd'hui afin de proposer des services pour traduire les adresses anciennes parisiennes en coordonnées géographiques (Cura et al. 2018) et combler des vides historiques des géocodeurs d'adresses modernes ${ }^{20}$.

Avec l'essor de la science ouverte et les obligations de rendre les données FAIR (findable, accessible, interoperable, reusable), la sémantisation des référentiels géohistoriques devient aussi une obligation lorsqu'une équipe de recherche crée des données géohistoriques. Ce travail permet d'ouvrir les usages des données notamment au-delà du SIG et pour ce faire, les normes et technologies du Web des données sont incontour- 
nables. Depuis plusieurs années, nous observons l'émergence de répertoires (gazetteers) de noms de lieux modélisés avec une approche orientée graphes, la première ressource de ce type étant vraisemblablement Pleiades ${ }^{21}$. Il s'agit d'un graphe collaboratif des noms de lieux du monde antique. Le réseau Pelagios ainsi que le World Historical Gazetteer $\left(\mathrm{WHG}^{22}\right.$ ) poursuivent ce travail et élargissent le périmètre pour inclure de nouvelles époques et différentes zones du monde. Par exemple, Jiménez-Badillo et al. (2020) ont constitué le premier index sémantisé de noms de lieux du Mexique colonial, en dépouillant de nombreuses sources textuelles historiques. Ces données seront aussi accessibles dans le WHG, grâce à un modèle pivot de partage de répertoires historiques, nommé Linked Places ${ }^{23}$. L'atelier Condorcet, "Référentiels géohistoriques sémantisés pour les humanités " $\left(2017-2019^{24}\right)$, a été un espace de réflexion et de travail sur la création et la diffusion des répertoires historiques de noms de lieux sur le Web des données. Ce type d'index constitue autant de ressources essentielles pour permettre, notamment, la reconnaissance et la résolution automatique des entités spatiales nommées dans des textes historiques et littéraires. Ce travail se poursuit dans le contexte du consortium PTM.

Aujourd'hui, la perspective de rendre les modèles et données FAIR en géohistoire est au cœur des travaux du consortium DataforHistory ${ }^{25}$, qui est porté par l'axe de recherche en histoire numérique du Laboratoire de recherche historique Rhône-Alpes (UMR 5190 LARHRA) à Lyon. Ce groupe de chercheurs mène un travail collaboratif depuis plusieurs années, visant à étendre le modèle conceptuel de référence développé par le Comité international pour la documentation (CIDOC), couramment abrégé CIDOC-CRM ${ }^{26}$, pour modéliser les données en histoire (Beretta 2021). Issu du monde des musées, CIDOC-CRM concerne à l'origine l'information relative au patrimoine culturel et a été utilisé avec succès par le projet européen PARTHENOS ${ }^{27}$, entre autres. Ce modèle cherche à atteindre les données du monde des bibliothèques, des archives et des institutions de recherche ${ }^{28}$. Il a été étendu, par exemple, par un usage en archéologie dans ARIADNE ${ }^{29}$. Pour étendre ce modèle avec une composante géohistorique, le gestionnaire $\mathrm{OntoMe}^{30}$ est une pièce-clé car il permet à la communauté de créer des modélisations partagées sous forme d'ontologies sur la base de CIDOC-CRM comme modèle pivot. L'expérience Symogih ${ }^{31}$, le premier système d'information historique avec une approche sémantique mise en œuvre par le LARHRA, est le point de départ du travail aujourd'hui mené par DataforHistory.

Les contributions présentées ci-dessous s'inscrivent pleinement dans le prolongement de ces grands travaux en SIG-Histoire. L'intégration de plans anciens par traitement automatique de sources historiques hétérogènes ou l'intégration de données historiques sémantisées en ligne interrogent toujours l'objectif d'interopérabilité et d'analyse historique. Ainsi, dans la deuxième partie de ce numéro, Isabella di Lenardo, Raphaël Barman, Federica Pardini et Frédéric Kaplan ainsi que Jean-Michel Follin, Élisabeth Simonetto et Anthony Chalais proposent de nouvelles méthodes statistiques et d'apprentissage profond pour l'extraction des données géographiques à partir des plans anciens, ici le cadastre dit napoléonien. Juliette Morel ainsi que Paul Lecat, Émile Blettery, Lætitia Delavoipière, Frédéric Saly-Giocanti, Sylvaine Conord, Valérie GouetBrunet, Alexandre Devaux, Mathieu Brédif et Frédéric Moret proposent 
une analyse en histoire urbaine mobilisant des sources historiques hétérogènes, et parfois littéraires, pour étudier respectivement l'histoire de deux villes françaises, Fort-de-France et Nanterre. Enfin, Christine Plumejeaud-Perreau, Mélissa Mimouni, Alain Bouju, Christian Pfister-Langanay, Thierry Sauzeau et Silvia Marzagalli ainsi que Landy Rajaonarivo, Marie-Noëlle Bessagnet, Christian Sallaberry, Annig Le Parc Lacayrelle et Philippe Roose s'approprient des normes et technologies du Web des données, pour diffuser et valoriser des données géohistoriques et patrimoniales.

\section{Systèmes d'information géographique et archéologie}

Intimement liée au développement de l'utilisation de l'archéométrie numérique (tachéomètres puis stations totales sur les champs de fouille), de la photographie aérienne et de terrain (d'abord scannée puis directement acquise au format numérique) et des SIG (pour le stockage et la représentation des données à références spatiales), l'analyse spatiale des données numériques en archéologie est maintenant devenue un outil incontournable de la production et de l'analyse de connaissances dans cette discipline. Il a donc été décidé de consacrer une partie entière de ce numéro spécial à l'information spatiale en archéologie en y proposant un recueil de projets et d'études de cas issus à la fois du monde académique et de l'archéologie préventive.

L'utilisation de l'informatique en archéologie remonte, comme pour à peu près toutes les sciences humaines et sociales, aux années 1950, lorsque l'on prit conscience du potentiel de cette nouvelle technologie pour le stockage et l'analyse statistique des informations ${ }^{32}$. Nous sommes ici à l'origine des bases des données! Dans le contexte de ces premiers balbutiements de la relation entre archéologie et informatique, naît, en 1965, la Newsletter of Computer Archaeology, toute première revue consacrée à la question, publiée par le département d'anthropologie de l'université d'État de l'Arizona (jusqu'en 1979). Sa diffusion fut, de prime abord, assez confidentielle et il fallut attendre le début des années 1970 ainsi que la "révolution " micro-informatique pour que l'on voie une première démocratisation de l'approche informatique en archéologie, de même que l'organisation de manifestations scientifiques sur la question. Parmi les événements à retenir, mentionnons la réunion fondatrice de l'association internationale Computer Applications and Quantitative Methods in Archaeology $\left(\mathrm{CAA}^{33}\right)$, qui se tint à Birmingham en 1973. Autre point tournant important : 1984, qui vit le lancement du premier grand périodique sur le sujet, Archaeological Computing Newsletter, par le département d'informatique de l'École polytechnique du North Staffordshire, en association avec l'Institut d'archéologie de l'université d'Oxford. De 2004 à 2007, la revue devint un supplément bisannuel d'Archeologia e Calcolatori $^{34}$, créée, quant à elle, en 1990 à l'université de Sienne et maintenant publiée par le CNR italien ${ }^{35}$. Depuis ces moments fondateurs, les manifestations et les périodiques, de même qu'avec le développement d'Internet à partir des années 1990, les projets en ligne de toutes sortes se sont évidemment multipliés, dans toutes les langues. Compte tenu du fait qu'Humanités numériques est une revue francophone, men- 
tionnons encore le périodique Archéologues et ordinateurs, qui fut publié de 1982 à 1995 par le Centre de recherches archéologiques du CNRS à Valbonne Sophia Antipolis.

La prise en considération des contextes de la découverte des sources en archéologie étant fondamentale du point de vue méthodologique, cette discipline a toujours fait un usage extensif des apports de la géographie et de la cartographie. On comprendra donc aisément que l'archéologie, qui entretient un rapport privilégié à la notion d'espace dans son questionnement problématisé ${ }^{36}$, fut l'une des premières sciences historiques à avoir perçu l'intérêt des outils de la géomatique pour l'interprétation spatiale des bases de données (Conolly et Lake, 2012), et cela dès la deuxième moitié des années 1980, lorsque les premiers logiciels de SIG pour ordinateurs personnels furent disponibles (par exemple, Mapping Display and Analysis System [MIDAS], qui devint ultérieurement MapInfo). Si l'utilisation initiale de cette technologie répondait essentiellement au besoin de placer les artéfacts ou les fouilles sur de " simples " cartes, l'usage se complexifia avec le temps, jusqu'à devenir, pour les archéologues, un véritable outil de prospection et d'analyse, permettant de poser des questions croisées complexes, notamment, si l'on reprend les seules catégories retenues par Barge et al. (2004:19-20) : la mesure de la géométrie des objets, l'interpolation à l'ensemble d'un espace, la modélisation par le croisement des variables topographiques et données environnementales, le calcul des distances ou, encore, les études de contexte. Parallèlement à ce développement de l'utilisation de la géomatique par l'archéologie, il n'est pas inintéressant de préciser que cette dernière s'appropria aussi pleinement la conception et le dessin assistés par ordinateur (CAO et DAO, en anglais $C A D$ ), comme complément au dessin traditionnel. La télédétection, aérienne à partir des années 1900 (Campana 2017 ; Luo et al. 2019), puis satellitaire à très haute résolution spatiale à partir des années 2000 (Lasaponara et Masini 2005 ; Morehart 2012 ; Luo et al. 2019), et enfin par drone à partir des années 2010 (Koehler 2013 ; Campana 2017 ; Luo et al. 2019) a également rejoint les outils numériques de l'archéologie depuis l'invention du scanner dans les années 1950 et de la possibilité de géoréférencer des images numérisées dans les SIG et d'autres outils de géovisualisation 2D ou 3D, ou d'analyse spatiale et de géostatistiques. En effet, la géovisualisation 2D et la modélisation 3D par photogrammétrie (Peterson 1957 ; Fabbri et al. 2021) ou par scan 3D (Fabbri et al. 2021) font pleinement partie de la «boîte à outils " des archéologues.

En 2004, dans un article qui se présentait à la fois comme un état des lieux de l'utilisation des SIG par les archéologues français, et comme une sorte de plaidoyer pour un développement encore plus important de ladite utilisation, Barge et al. (2004: 16) faisaient remarquer que le monde anglo-saxon avait adopté les outils de la géomatique "de manière précoce par rapport à la pratique française, en archéologie [...], comme en général en sciences humaines ". Les mêmes auteurs font remarquer que les programmes européens Archaeomedes I et II, qui, entre 1992 et 1999, furent consacrés à l'étude de la désertification des régions méditerranéennes de l'Europe, de l'Antiquité à nos jours, étaient parmi les seuls, il y a dix-sept ans de cela, à avoir fait l'objet d'une publication formelle, en signalant, au passage, que cette dernière était probablement le fait de la participation des géographes (Barge et al. : 17). Les temps ont bien chan- 
gé et il suffit de consulter le répertoire des projets sur le site geoPratiq ${ }^{37}$ qui est l'observatoire des pratiques géomatiques en sciences historiques, porté par le centre de recherche Archéologie et sciences de l'Antiquité (UMR 7041 ArScAn). En outre, les grands opérateurs en archéologie préventive, l'INRAP en premier lieu, n'hésitent plus aujourd'hui à faire appel aux techniques de la géomatique dans leurs opérations quotidiennes.

Le reste de la francophonie suivit à peu près l'évolution qui a été celle de la France en matière d'application des SIG à l'archéologie, même s'il ne faut surtout pas caricaturer au point de ne pas reconnaître les différences nationales et de convenir d'un seul schéma pour tous les pays concernés. Pour illustrer le phénomène par un seul exemple, notons la singularité de l'expérience québécoise, en raison : (1) du caractère mixte de son approche en archéologie, à la fois influencée par le modèle anglosaxon et par le modèle français ; (2) de l'expertise canadienne en matière de géomatique (le Canada a été pionnier dans le domaine, avec son Canada Geographic Information System, créé au début des années 1960) ; (3) de la nature profondément différente des terrains et objets d'études en France et au Québec (ce qui mena d'ailleurs au développement de deux modèles pas tout à fait similaires en archéologie préventive). En ce sens, si la France a multiplié plus rapidement les déploiements de SIG archéologiques sur des contextes précis (la TGIR Huma-Num du CNRS constitue l'une des solutions d'hébergement pérenne, en France, de cette masse d'information et son consortium MASA ${ }^{38}$ fédère de nombreuses initiatives dans ce sens), on peut dire que le Québec s'est, dans un premier temps, davantage concentré sur le développement des outils, des méthodologies et des applications, en collaboration avec les facultés de géographie et d'ingénierie.

Quoi qu'il en soit, la globalisation actuelle des échanges académiques et universitaires ne permet plus vraiment ce type d'observation à l'échelle des États. Plusieurs WebSIG visent d'ailleurs à cumuler, voire à croiser les bases de données sur des contextes ou des thématiques similaires, plutôt que de chercher à "nationaliser " la recherche. Parmi ces WebSIG, mentionnons, par exemple, les projets ArkeoGIS ${ }^{39}$ et Chronocarto $^{40}$, le premier des deux étant basé au centre de recherche Archéologie et histoire ancienne : Méditerranée-Europe (UMR 7044 ARCHIMÈDE) et étant initialement une entreprise de partage de données entre chercheurs français et allemands sur la vallée du Rhin en un même module, le second étant un portail du laboratoire Archéologie et philologie d'Orient et d'Occident (UMR 8546 Aoroc) visant à regrouper divers projets et à leur offrir des outils sur mesure, en fonction de leurs besoins (échelle du SIG, type de base de données, etc.).

La diversité des questions de recherche et des formats des SIG que l'on retrouve dans ces WebSIG, comme dans d'autres, sont d'ailleurs parfaitement illustrés par les cinq contributions qui composent la troisième partie du présent numéro thématique : Francis Tassaux fait le bilan problématisé des deux grands projets d'atlas archéologiques informatisés, respectivement sur la côte Adriatique et sur les Balkans occidentaux dans l'Antiquité tardive et au haut Moyen Âge, qu'il a dirigé ; Nicolas Revert nous fait part de la conception, des résultats et des limites d'un SIG qui a pour objectif archéologique d'établir les paramètres d'emplacement des établissements ruraux de la Somme à l'époque romaine ; Élodie Guillon et Antoine Laurent présentent le SIG du projet ERC Mapping An- 
cient Polytheisms (EA 4601 PLH, université Toulouse-Jean-Jaurès) ; Christophe Tufféry, Vincent Delvigne, Paul Fernandes et Céline BressyLéandri s'intéressent aux conditions de constitution de lithothèques avec les outils de la géomatique ; Nicolas Poirier dévoile les premiers résultats du programme REPERAGE (UMR 5608 THRACE) sur les dynamiques d'occupation de la vallée de la Garonne sur la très longue durée, mais afin de répondre à un questionnement historique axé sur l'Antiquité tardive et le haut Moyen Âge. Une seule constante ici : les cinq articles portent principalement sur des époques relativement anciennes, de la Préhistoire au Moyen Âge. Évidemment, des périodes plus récentes auraient pu aussi parfaitement trouver leur place dans la présente section, l'archéologie s'étant déjà ouverte depuis un certain temps aux époques moderne et, même, contemporaine.

\section{Conclusion}

La diversité des sujets abordés illustre bien la vitalité actuelle des humanités numériques spatialisées. L'enjeu de ce numéro n'était toutefois pas de tirer un bilan exhaustif de cette problématique, mais plutôt d'amener les spécialistes de différentes disciplines en SHS à entrer dans un dialogue sur les différentes approches mobilisées et les méthodes d'analyse des données spatiales mises en œuvre à l'ère du numérique.

En proposant un tel thème pour le troisième numéro d'Humanités numériques, nous avions pour objectif de partager des pratiques numériques pluridisciplinaires en SHS, en nous appuyant à la fois sur les résultats de projets aboutis et sur des travaux de recherche émergents. Les humanités numériques spatialisées, telles que conçues ici, prennent pleinement en considération les aspects méthodologiques d'une recherche appliquée ainsi que ses questionnements spécifiques disciplinaires.

REMERCIEMENTS. Le résultat final n'aurait évidemment pas été aussi satisfaisant sans la pleine collaboration du comité de direction de la revue, ainsi que des évaluateurs et évaluatrices du présent numéro. Qu'il nous soit permis de les remercier ici. Les éditeurs invités en profitent aussi pour remercier toutes les autres personnes qui ont participé de près ou de loin à ce beau numéro, principalement Florence Daniel, éditrice à la Maison des sciences de l'homme en Bretagne. Nous remercions également Bertrand Dumenieu d'avoir partagé avec nous les détails historiographiques de la mise en ligne de la carte Cassini.

\section{Bibliographie}

Aron, Paul, Laurence Brogniez, Tatiana Debroux, Jean-Michel Decroly et Christophe Loir. 2017. "À la recherche des chronotopes du roman urbain. Une cartographie des Mystères de Bruxelles (1845-1846)». Mappemonde 121. https://doi.org/10.4000/mappemonde.3592.

Bakhtine, Mikhaïl. 1978. Esthétique et théorie du roman. Paris : Gallimard.

Barge, Olivier, Xavier Rodier, Gourguen Davtian et Laure Saligny. 2004. "L'utilisation des systèmes d'information géographique appliquée à l'archéologie française ". Revue d'archéométrie 28 : 15-24. https://doi.org/10.3406/arsci.2004.1058. 
Beretta, Francesco. 2021. «A Challenge for Historical Research : Making Data FAIR Using a Collaborative Ontology Management Environment (OntoME) ». Semantic Web 12 (2) : 279294. https://doi.org/10.3233/SW-200416.

Bernard, Catherine, Marc Comelongue, Louise-Emmanuelle Friquart, Laure Krispin, Géraud de Lavedan et Laure Moreau. 2012. "Urban-Hist, le patrimoine toulousain à la carte ». Les Cahiers de Framespa 10. https://doi.org/10.4000/framespa.1459.

Bodenhamer, David J., John Corrigan et Trevor M. Harris, éd. 2010. The Spatial Humanities. GIS and the Future of Humanities Scholarship. Bloomington et Indianapolis : Indiana University Press. http://www.jstor.org/stable/j.ctt16gzj77.

Bretagnolle, Anne, Timothée Giraud et Nicolas Verdier. 2010. « Modéliser l'efficacité d'un réseau. Le cas de la poste aux chevaux dans la France pré-industrielle (1632-1833) ». L'Espace géographique 39 (2) : 117-131. https://doi.org/10.3917/eg.392.0117.

Campana, Stefano. 2017. " Drones in Archaeology. State-of-the-art and Future Perspectives ». Archaeological Prospection 24 (4), 275-296. https://doi.org/10.1002/arp.1569.

Dominguès, Catherine, Laurence Jolivet, Carmen Brando et Marion Cargill. 2019. "Place and Sentiment-based Life Story Analysis. From the Spanish Republican Army to the French Resistance ». Revue française des sciences de l'information et de la communication 17. https://doi.org/10.4000/rfsic.7228.

Conolly, James et Mark Lake. 2012. Geographical Information Systems in Archaeology. Cambridge : Cambridge University Press.

Costa, Laurent. 2012. "La construction de référentiels géohistoriques : un enjeu pour l’interdisciplinarité dans les sciences historiques ". L'Espace géographique 41 (4) : 340-351. http s://doi.org/10.3917/eg.414.0340.

Cura, Rémi, Bertrand Dumenieu, Nathalie Abadie, Benoît Costes, Julien Perret et Maurizio Gribaudi. 2018. « Historical Collaborative Geocoding ». ISPRS International Journal of GeoInformation 7 (7) : 262. https://doi.org/10.3390/ijgi7070262.

Donaldson, Christopher, Ian Gregory et Patricia Murrieta-Flores. 2015. " Mapping "Wordsworthshire" : A GIS Study of Literary Tourism in Victorian Lakeland ». Journal of Victorian Culture 20 (3) : 287-307. https://doi.org/10.1080/13555502.2015.1058089.

Dumenieu, Bertrand, Julien Chadeyron, Pascal Cristofoli, Julien Perret, Laurence Jolivet, Stéphane Baciocchi, Stéphane Gomis, Maurozio Gribaudi, Isabelle Langlois, Claude Motte et Marie-Christine Vouloir. 2019. " Engraved Footprints from the Past. Retrieving Cartographic Geohistorical Data from the Cassini Carte de France, 1750-1789 ». Poster présenté à l'International Cartographic Conference 2019. Tokyo, 15 juillet. Données : https://doi.org/10 .7910/DVN/XP8J6P.

Eiteljorg, Harrison. 2004. « Computing for Archaeologists ». Dans A Companion to Digital Humanities, édité par Susan Schreibman, Ray Siemens et John Unsworth. Oxford : Blackwell Publishing. http://www.digitalhumanities.org/companion/view?docId=blackwell/9781405103213/9781405103213.xml\&chunk.id=ss1-2-2\&toc.depth=1\&toc.id=ss1-2-2\& brand $=$ default.

Fabbri, Stefano, Veronica Chiarini, Massimo Ercolani, Garibaldi Sansavini, Tommaso Santagata, Jo De Waele. 2021. «Terrestrial Laser Scanning, Geomorphology and Archaeology of a Roman Gypsum Quarry (Vena del Gesso Romagnola Area, Northern Apennines, Italy) ». Journal of Archaeological Science : Reports 36 (4) : 102810. https://doi.org/10.1016/j.jasrep.2021.102810.

Frontini, Francesca, Carmen Brando, Marine Riguet, Clémence Jacquot et Vincent Jolivet. 2016. «Annotation of Toponyms in TEI Digital Literary Editions and Linking to the Web of Data ». MATLIT : Materialidades da Literatura 4 (2) : 49-75.

Frontini, Francesca, Carmen Brando, Joanna Byszuk, Ioana Galleron, Diana Santos et Ranka Stanković. 2020. « Named Entity Recognition for Distant Reading in ELTeC ». Dans Proceedings of CLARIN Annual Conference 2020. Virtual Edition. Madrid, 5-7 October, édité par Costanza Navarretta et Maria Eskevich. Madrid : CLARIN. https://www.clarin.eu/clarin-an nual-conference-2020-abstracts.

Gregory, Ian et Paul Ell. 2007. Historical GIS. Technologies, Methodologies, and Scholarship. Cambridge : Cambridge University Press. https://doi.org/10.1017/CBO9780511493645.

Jiménez-Badillo, Diego, Patricia Murrieta-Flores, Bruno Martins, Ian Gregory, Mariana Favila-Vázquez et Raquel Liceras-Garrido. 2020. "Developing Geographically Oriented NLP Approaches to Sixteenth-Century Historical Documents : Digging into Early Colo- 
nial Mexico ». Digital Humanities Quarterly 14 (4). http://www.digitalhumanities.org/dhq/vol/14/4/000490/000490.html.

Kergosien, Éric, Pierre Maurel, Mathieu Roche et Maguelonne Teisseire. 2015. «SENTERRITOIRE pour la détection d'opinions liées à l'aménagement d'un territoire ». Revue internationale de géomatique 25 (1) : 11-34. https://doi.org/10.3166/RIG.25.11-34.

Knowles, Anne Kelly. 20oo. Introduction. Special Issue « Historical GIS : The Spatial Turn in Social Science History ». Social Science History 24 (3) 451-470.

Koehler, Alain. 2013. «Les drones en archéologie ». Dans L’Archéologie au laboratoire, édité par Stéphanie Thiébault et Pascal Depaepe, 233-241. Paris : La Découverte.

Lasaponara, Rosa et Nicola Masini. 2005. « QuickBird-based Analysis for the Spatial Characterization of Archaeological Sites : Case Study of the Monte Serico Medieval Village ». Geophysical Research Letters 32 (12) : L12313. https://doi.org/10.1029/2005GL022445.

Luo, Lei, Xinyuan Wang, Huadong Guo, Rosa Lasaponara, Xin Zong, Nicola Masini, Guizhou Wang, Pilong Shi, Houcine Khatteli, Fulong Chen, Shahina Tariq, Jie Shao, Nabil Bachagha, Ruixia Yang, Ya Yao, 2019. " Airborne and Spaceborne Remote Sensing for Archaeological and Cultural Heritage Applications : a Review of the Century (1907-2017)». Remote Sensing of Environment 232 : 111280, https://doi.org/10.1016/j.rse.2019.111280.

McDonough, Katherine, Ludovic Moncla et Matje van de Camp. 2019. "Named Entity Recognition Goes to Old Regime France : Geographic Text Analysis for Early Modern French Corpora ». International Journal of Geographical Information Science 33 (12) : 2498-2522. http s://doi.org/10.1080/13658816.2019.1620235.

Minard, Charles Joseph. 1869. Carte figurative des pertes successives en hommes de l'armée française dans la campagne de Russie 1812-13. Paris : Régnier et Dourdet.

Moncla, Ludovic, Mauro Gaio, Javier Nogueras-Iso et Sébastien Mustière. 2016. « Reconstruction of Itineraries from Annotated Text with an Informed Spanning Tree Algorithm ». International Journal of Geographical Information Science 30 (6) : 1137-116o. https://doi.org/1 0.1080/13658816.2015.1108422.

Morehart, Christopher T. 2012. " Mapping Ancient Chinampa Landscapes in the Basin of Mexico : a Remote Sensing and GIS Approach ». Journal of Archaeological Science 39 (7) : 2541-2551. https://doi.org/10.1016/j.jas.2012.03.001.

Moretti, Franco. 1999. Atlas of the European Novel, 180o-190o. Londres : Verso.

Moretti, Franco. 200o. « Conjectures on World Literature ». New Left Review 1. https://newl eftreview.org/issues/iil/articles/franco-moretti-conjectures-on-world-literature.

Moretti, Franco. 2007. « Cartes ». Romantisme 138 (4) : 11-34. https://doi.org/10.3917/rom.13 8.0011.

Murrieta-Flores, Patricia et Bruno Martins. 2019. "The Geospatial Humanities : Past, Present and Future ». International Journal of Geographical Information Science 33 (12): 2424-2429. https://doi.org/10.1080/13658816.2019.1645336.

Noizet, Hélène, Boris Bove, Laurent Costa, éd. 2013. Paris de parcelles en pixels. Analyse géomatique de l'espace parisien médiéval et moderne. Saint-Denis et Paris : Presses universitaires de Vincennes et Comité d'histoire de la Ville de Paris.

Perret, Julien, Maurizio Gribaudi et Marc Barthelemy. 2015. Roads and Cities of 18th Century France. Scientific Data 2 : 150048. https://doi.org/10.1038/sdata.2015.48.

Peterson, Charles E., 1957. « Photogrammetry for HABS ». Journal of the Society of Architectural Historians 16 (4) : 29. https://doi.org/10.2307/987875.

Piatti, Barbara, Hans Rudolf Bär, Anne-Kathrin Reuschel, Lorenz Hurni et William Cartwright. 2009. «Mapping Literature : Towards a Geography of Fiction ». Dans Cartography and Art, édité par William Cartwright, Georg Gartner, et Antje Lehn, 1-16. Berlin et Heidelberg : Springer. https://doi.org/10.1007/978-3-540-68569-2_15.

Pinol, Jean-Luc. 2019. Convois. La déportation des Juifs de France. Paris : Éditions du Détour.

Simon, Rainer, Elton Barker, Leif Isaksen et Pau de Soto Cañamares. 2015. « Linking Early Geospatial Documents, One Place at a Time : Annotation of Geographic Documents with Recogito ».e-Perimetron 10 (2) : 49-59. http://www.e-perimetron.org/Vol_10_2/Simon_et_al.pdf.

Snow, John. 1855. On the Mode of Communication of Cholera. Londres : John Churchill. 
Varet-Vitu, Anne, Mathieu Marraud et Éric Mermet. 2020. «Spatialités sociales à Paris à la veille de la Révolution. Les apports d'un système d'information géographique ». Histoire urbaine 58 (2) : 157-186. https://doi.org/10.3917/rhu.058.0159.

\section{Notes}

1 Comme l'illustrent les nombreux articles sur les thématiques comme « maps and mapping ", "spatio-temporal modeling, analysis and visualisation ", "geospatial analysis, interfaces and technology " repérables dans l'Index of Digital Humanities Conferences (https://dhabstracts.library.cmu.edu).

2 https://geohumanities.org.

3 L'atelier «HumaNS'2018 : Humanités numériques spatialisées » organisé par Carmen Brando, Francesca Frontini et Mathieu Roche, le 6 novembre 2018 à Montpellier, dans le cadre de la conférence SAGEO 2018. Voir http://psig.huma-num.fr/HumaNS/.

4 Les deux journées d'étude exploratoires « Archéologie, histoire et analyse spatiale. Dialogue interdisciplinaire sur la question des SIG archéologiques et historiques » organisées par Dominic Moreau, en collaboration avec Xavier Deru (UMR 8164 HALMA, université de Lille), Éric Masson et Pierre-Gil Salvador (ULR 4477 TVES, université de Lille), les 31 mai et $1^{\mathrm{er}}$ juin 2018 à Lille, grâce à la MESHS, en soutien à lémergence du projet DANUBIUS (http s://danubius.huma-num.fr) de l'UMR 8164 HALMA (université de Lille, CNRS, ministère de la Culture). Voir http://www.meshs.fr/page/archeologie_histoire_et_analyse_spatiale_dialogue_interdisciplinaire.

5 https://journals.openedition.org/revuehn/565/.

6 Voir les guidelines TEI spécifiques (https://tei-c.org/release/doc/tei-p5-doc/en/html/ND .html).

7 https://www.lancaster.ac.uk/mappingthelakes/.

8 https://recogito.pelagios.org.

9 https://cahier.hypotheses.org.

$10 \mathrm{https} / / /$ www.distant-reading.net.

11 https://distantreading.github.io.

12 https://rrchnm.org.

13 https://alpage.huma-num.fr/presentation/objectifs/.

$14 \mathrm{https} / /$ paris-timemachine.huma-num.fr/accueil/.

15 Ce chantier important a été piloté et réalisé par Marie-Christine Vouloir et Claude Motte, ingénieures du Laboratoire de démographie et d'histoire sociale (LaDéHiS, UMR $8558 \mathrm{CRH})$.

16 Voir l'historique ici : http://cassini.ehess.fr/fr/html/5_donnees.htm.

17 https://geohistoricaldata.org.

$18 \mathrm{http}: / /$ cassini.ehess.fr.

19 Les données de la base dite «Cassini » sont en cours de reprise et d'enrichissement par l'équipe GeoHistoricalData pour les diffuser dans des formats lisibles par les machines (interopérables).

20 https://adresse.data.gouv.fr.

21 https://pleiades.stoa.org.

22 http://whgazetteer.org.

23 https://github.com/LinkedPasts/linked-places/.

24 http://www.chartes.psl.eu/sites/default/files/atoms/files/referentiels-geohistoriques_2017-12-07.pdf.

$25 \mathrm{http} / / /$ dataforhistory.org.

$26 \mathrm{http}: / /$ www.cidoc-crm.org.

27 https://www.parthenos-project.eu.

28 https://doc.biblissima.fr/introduction-au-cidoc-crm/. 
29 https://ariadne-infrastructure.eu.

30 http://ontologies.dataforhistory.org.

31 http://symogih.org.

32 Sur l'histoire de l'informatique appliquée à l'archéologie, voir Eiteljorg, 2004, que l'on peut compléter par le site http://archaeologicalcomputing.lincei.it, qui est un projet de l'Accademia Nazionale dei Lincei (dernière modification : 2016). Pour l'état historique de la question, il a été décidé de ne pas citer d'autres références. On comprendra toutefois que la méthodologie et les techniques ont grandement avancé depuis et que leur constante évolution empêche de citer une référence définitive. En ce sens, contentonsnous, de manière inusitée, de renvoyer à une page Wikipédia, https://en.wikipedia.org/wiki/Computational_archaeology/, qui propose de nombreuses références bibliographiques et "sitographiques " sur le sujet, tout en étant elle-même en constante évolution.

33 https://caa-international.org.

34 http://www.archcalc.cnr.it/pages/newsletter.php.

35 http://www.archcalc.cnr.it.

36 «Les archéologues sont depuis longtemps concernés par l'espace, de deux manières :

- par la nature même de l'information qu'ils collectent : l'information archéologique est spatialisée,

- en tant que facteur d'explication de leurs découvertes et de leurs analyses. " (Barge et al. 2004)

37 http://pratiq.huma-num.fr/Observatoire/Projects/.

38 https://arear.huma-num.fr.

39 https://arkeogis.org.

40 https://www.chronocarto.eu.

\section{Auteurs}

\section{Carmen Brando}

UMR 8558 CRH, EHESS, Paris, France

Carmen Brando $(\mathrm{PhD})$ est ingénieure de recherche en humanités numériques à l'EHESS et membre du Centre de recherches historiques (UMR $8558 \mathrm{CRH}$ ). Elle est spécialiste des méthodes en traitement automatique des langues, du Web sémantique et de la géomatique appliquée aux sciences humaines et sociales.

ORCID 0000-0001-7098-3522

carmen.brando@ehess.fr

\section{Francesca Frontini}

Istituto di Linguistica Computazionale, CNR, Pise, Italie, et ERIC CLARIN

Francesca Frontini (PhD) est chercheuse à l'Institut de linguistique computationnelle du Consiglio Nazionale delle Ricerche (Italie) et membre du comité de pilotage de l'infrastructure européenne CLARIN. Ses intérêts de recherche portent sur les ressources et technologies du langage ; elle s'intéresse entre autres à l'annotation des éléments spatiaux dans les corpus numériques.

ORCID 0000-0002-8126-6294

francesca.frontini@ilc.cnr.it

\section{Dominic Moreau}

UMR 8164 HALMA, université de Lille, CNRS, ministère de la Culture, Lille, France

Dominic Moreau $(\mathrm{PhD})$ est maître de conférences à l'université de Lille et membre du laboratoire Histoire, archéologie, littérature des mondes anciens (UMR 8164 HALMA). Spécialiste de l'Antiquité tardive et de l'histoire du christianisme antique, il concentre principalement ses travaux actuels sur l'espace balkanique ; il dirige ainsi le projet $D A N U$ BIUS (ANR/I-SITE ULNE), codirige la Mission archéologique internationale à Zaldapa (Bulgarie) et porte le projet de réseau international HAEMUS pendant sa phase de mise en œuvre. 


\section{Mathieu Roche}

UMR 9000 TETIS, université de Montpellier, AgroParisTech, CIRAD, CNRS, INRAE, Montpellier, France

Mathieu Roche (PhD, HDR) est chercheur au CIRAD, au laboratoire Territoires, environnement, télédétection et information spatiale (UMR 900o TETIS). Il a coordonné de nombreux projets dans le domaine de la fouille de textes, en particulier des projets pluridisciplinaires en collaboration avec des linguistes, géographes et épidémiologistes. ORCID 0000-0003-3272-8568

mathieu.roche@cirad.fr

\section{Éric Masson}

ULR 4477 TVES, université de Lille, Lille, France

Éric Masson (PhD) est maître de conférences à l'université de Lille et membre du laboratoire Territoires, villes, environnement et société (ULR 4477 TVES). Ses recherches portent sur la géomatique appliquée aux questions environnementales dans une large perspective pluridisciplinaire (sciences sociales, géosciences, sciences de l'ingénieur). ORCID 0000-0003-2931-6175

eric.masson@univ-lille.fr

\section{Droits d'auteur}

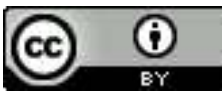

Les contenus de la revue Humanités numériques sont mis à disposition selon les termes de la Licence Creative Commons Attribution 4.0 International. 